\title{
Por que não recomendar estatinas como prevenção primária?
}

\author{
Charles Dalcanle Tesser, Armando Henrique Norman.
}

\section{RESUMO}

Este artigo tece uma crítica à mudança de postura e práticas médicas a respeito do uso de estatinas como prevenção primária (P1). Tal tema é de grande relevância clínica para a atenção primária à saúde e para a saúde pública. No Tratado de Medicina de Família e Comunidade (edição de 2018), o capítulo sobre P1 de doenças cardiovasculares assume implicitamente que as estatinas devem ser usadas como P1, sem discussão dos problemas envolvidos nisso. Este artigo faz uma síntese dos problemas e polêmicas envolvidos na prescrição de estatinas em P1 a partir de 2012, quando uma primeira metanálise recomendou esse uso. Discutimos criticamente as evidências a respeito, a partir de clássicos critérios éticos e técnicos. Defendemos que a evidência para usar estatinas como P1 é muito fraca e não confiável, frente a danos potenciais significativos e benefícios muito pequenos. Mais pesquisas são necessárias, mais transparentes e com menos influência da indústria farmacêutica.

Revista da Rede APS 2019

Publicada em:

22/03/2019

Charles Dalcanle Tesser (UFSC),

Armando Henrique Norman (UFSC).

Correspondência para: Charles Dalcanle Tesser, charles.tesser@ufsc.br

Palavras-chave: Atenção primária à saúde; Prevenção primária; Prevenção quaternária; Doenças Cardiovasculares; Fármacos Cardiovasculares. 


\section{INTRODUÇÃO}

Este artigo foi suscitado pela segunda edição revisada, em 2018, de um dos dois principais livros nacionais de referência para os profissionais da atenção primária à saúde (APS), o "Tratado de medicina de família e comunidade" (1). Assim como seu par, "Medicina ambulatorial: condutas de atenção primária baseadas em evidências" (2), esse livro trata de temas clínicos e sanitários de relevância para a saúde coletiva. Um deles é a prescrição de fármacos visando reduzir o risco cardiovascular em pessoas sem lesão cardiovascular e assintomáticas, ou seja, como prevenção primária (P1). O uso de estatinas como P1 é crônico e tem grande impacto na rotina dos serviços $e$ profissionais da APS e também na vida dos usuários. Mais de um quarto dos norte-americanos maiores de 40 anos usa alguma estatina (3).

Na saúde coletiva e na medicina de família e comunidade brasileira, pouca discussão e pesquisa sobre este tipo de intervenção estão publicadas. Uma busca no portal scielo (http://www.scielo.org/php/index.php), sem restrição de tempo, em todos os índices (em 04/12/2019), com os descritores combinados 'Prevenção Primária' e 'Doenças Cardiovasculares', resultou em 47 artigos, 35 brasileiros. Nenhum destes 35, avaliados pelos títulos, se aproxima do problema da indicação de uso das estatinas como P1. Já no portal LILACS (http://lilacs.bvsalud.org/) encontrou-se 138 documentos. Destes, apenas 14 se aproximam do tema e somente um é da saúde coletiva (não abordando as estatinas). Desses 14 artigos, 10 não abordam o tema, dois reafirmam a recomendação de estatinas para P1 e dois a abordam criticamente (4), sendo um uma carta ao editor (5) advertindo para o perigo do excesso de medicalização e iatrogenia não compensadas por benefícios substantivos.

Na 1a edição (de 2012) do Tratado de Medicina de Família e Comunidade, o capítulo "Prevenção primária e secundária para doenças cardiovasculares" afirma nos highlights iniciais que "não há evidência de uso de qualquer medicação como prevenção primária para doença cardiovascular" (6:1233). Na edição de 2018, no mesmo capítulo, de outros autores (7), não há menção às estatinas nos highlights, porém no texto aparece (7:1337): "A medicação de escolha para a prevenção primária de eventos cardiovasculares é a estatina". Não há um único comentário sobre a mudança de recusar a idéia do uso de estatinas em P1 para (implicitamente) aceitar essa idéia. Os autores apenas reconhecem não haver consenso sobre como e quando indicá-las em P1, considerando quatro diretrizes clínicas (National Institute for Health and Care Excellence - NICE; da Sixth Joint Task Force of the European Society of Cardiology; da United States Preventive Services Task Force - USPSTF; e a I Diretriz Brasileira de Prevenção Cardiovascular - da Sociedade Brasileira de Cardiologia). O capítulo parece considerar, subliminarmente, de modo positivo e confiável, natural que o profissional recomende e/ou prescreva estatinas como P1 em certas situações.

O objetivo deste artigo é discutir criticamente essa mudança do posicionamento de muitos médicos e do Tratado sobre a prescrição de estatinas como P1, e defender uma atitude oposta: a da manutenção de que não se 'deve' recomendar estatinas como P1. Inicialmente, apresentamos e discutimos os principais estudos e polêmicas sobre o uso de estatinas como P1. A seguir, sintetizamos os critérios éticos e técnicos norteadores das decisões envolvendo recomendações desses tratamentos preventivos. Por último, aplicamos tais critérios ao problema em questão.

\section{A PROBLEMÁTICA DAS ESTATINAS}

Dada o grande volume de literatura, optamos por realizar uma síntese livre dos principais aspectos relativos ao uso de estatinas como P1. Em 2012, foi publicada a metanálise sobre o uso das estatinas como P1 do Cholesterol Treatment Trialists (CTT) Collaborators (8), que concluiu que havia benefícios para pessoas sem doença cardiovascular prévia para a utilização de estatinas como P1, com mínimos danos.

Em 2013, uma revisão da Colaboração Cochrane (9) concluiu que as estatinas "reduzem a mortalidade por todas as causas e os eventos cardiovasculares, sem aumentar o risco de eventos adversos, entre as 
pessoas com baixo risco cardiovascular (RCV) inferior a $10 \%$ em dez anos" (10).

Todavia, o BMJ publicou, também em 2013, uma reavaliação independente da metanálise do CTT que contrariou suas conclusões (11): encontrou que em pessoas com baixo RCV $(<20 \%$ nos próximos 10 anos), o uso de estatinas não está associado a redução do risco global de morte ou doença séria, e 140 pessoas de baixo risco devem usar estatinas por 5 anos para prevenir um ataque cardíaco ou acidente vascular cerebral. Concluiu que qualquer dano das estatinas desequilibraria a equação no sentido negativo (10). Esta reavaliação foi criticada publicamente pelo diretor do CTT e foi nomeado um painel independente pelo BMJ, que decidiu por unanimidade contra o pedido de retratação, sendo sua publicação mantida (12).

Mesmo assim, instituições respeitadas começaram a recomendar o uso de estatina como $\mathrm{P} 1$. $\mathrm{O}$ apoio a essa diretriz pelo NICE (13) inglês e pelos cardiologistas norte-americanos (14) gerou em 2014 vários milhões de prescrições (10). Em 2015, o NICE aprovou proposta de introdução de pagamento para os General Practitioners que prescrevessem estatinas para os pacientes com $10 \%$ de RCV (15).

Um dos graves problemas da metanálise do CTT é a opacidade completa dos seus dados primários. Sobre os dados de eficácia, o CTT concordou que sua metanálise ao nível dos pacientes seria realizada em estrita confiança, o que significa que não seria compartilhada sem a permissão de quem realizou os estudos (16). Assim, o CTT teve acesso exclusivo aos dados primários dos pacientes sobre eficácia, sem permitir avaliação por peritos independentes. Apesar dos pedidos por muitos anos, os dados primários nunca foram socializados para a comunidade científica (17).

Quanto aos dados sobre danos das estatinas, nem os pesquisadores do CTT nem pesquisadores independentes tiveram acesso aos dados primários. O CTT só recebeu dados de eventos adversos de nível de paciente sobre o câncer e as razões para a interrupção do tratamento. Os efeitos adversos citados na metanálise da CTT baseia-se em relatórios publicados e não em dados primários
(10). Quais seriam os supostos benefícios e os danos do uso de estatina como P1?

\section{BENEFÍCIOS POTENCIAIS}

Diamond (18) discute os benefícios das estatinas em P1 usando alguns ensaios clínicos, e os mostra como devem ser apresentados: por meio da redução do risco absoluto. Usando dados do ensaio ASCOTT-LLA, Diamond (18) mostra que o benefício é mínimo ( $1,1 \%$ de redução do risco absoluto). Tal benefício só aparenta ser clinicamente significativo se for valorizada isoladamente a redução do risco relativo, que é de $36 \%$ para o desfecho composto 'infarto não fatal + doença coronariana fatal'. Outra metanálise (19) focada na redução da mortalidade, demonstrou que o benefício da estatina na P1 seria de atrasar entre 5 e 19 dias a morte; e entre 10 a 27 dias em quem tem doença cardiovascular. Não há polêmica sobre isso, apenas uso propagandístico da redução do risco relativo de $36 \%$. O benefício é simplesmente muito pequeno.

\section{DANOS POTENCIAIS}

Uma primeira cautela sobre danos é que vários dos ensaios clínicos contém um período inicial em que os pacientes recebem a droga, mas o estudo não começou formalmente (período de run-in). 0 efeito do run-in tem exclui na fase pré-inicial dos estudos até $26 \%$ de todos os indivíduos elegíveis após uso de sinvastatina por 1 mês (18). É razoável argumentar que estes estudos não poderiam fornecer dados confiáveis sobre danos (mesmo que haja evasão também no grupo controle), a não ser que existam dados primários acessíveis sobre o porquê dessa eliminação (o que não há); mas eles são incluídos nas metanálises.

Além disso, os ensaios frequentemente excluem os indivíduos com maior risco de efeitos adversos, como pacientes com doença hepática, insuficiência renal, fragilidade geral, polifarmácia e idosos - o que pode chegar a 30\% dos inicialmente elegíveis (20).

Adicionalmente, o abandono de voluntários dos estudos resulta em subestimação dos efeitos adversos: cerca de $10 \%$ dos pacientes abandonam os estudos por várias razões. Esse grupo pode representar justamente aqueles pacientes que 
sofreram os mais significativos efeitos adversos (20).

Diamond (18) aponta alguns danos potenciais tais como aumento da taxa de câncer, catarata, diabetes, comprometimento cognitivo e distúrbios musculoesqueléticos. Vários deles aparecem nas metanálises e outros não. A atuação das estatinas na fisiologia é muito complexa, com ações mutuamente contraditórias, além de haver contradição entre evidências tanto de ensaios clínicos quanto de estudos observacionais (20).

Em relação ao aumento dos cânceres, a maioria dos ensaios com estatinas em P1 dura entre 2 a 5 anos, pouco tempo para se desenvolver a maioria dos cânceres na população mais jovem. Alguns ensaios mostraram maior incidência ou mortalidade por câncer (18), mas há ausência desse achado nas grandes revisões (CTT e Cochrane), que quando mencionam esse problema o atribuem a cânceres pré-existentes (9). Há plausibilidade biológica (18) e evidências sobre esse efeito adverso, que parece maior em idosos (21): baixos níveis plasmáticos de colesterol LDL estão associados a um risco aumentado de câncer (22,23). Há evidências observacionais a favor e contra a presença desse efeito adverso $(20,24,25)$. O maior ensaio clínico com idosos mostrou aumento de mortalidade por câncer, o qual anulou o efeito da diminuição da mortalidade cardiovascular, mantendo igual a mortalidade geral (26). Mais estudos são necessários e a dúvida persiste.

A miopatia é o efeito adverso mais comum das estatinas, mais comum em mulheres e idosos, porém foi relatada nos ensaios em cerca de apenas 1\% dos pacientes, por várias razões: (a) há exclusões de pacientes nas fases iniciais dos ensaios, que podem chegar até 30 ou $35 \%$ dos pacientes elegíveis (27); (b) o critério para seu diagnóstico é um aumento de mais de 10 vezes de creatina quinase, sendo que abaixo disso há ampla subnotificação, e a maioria dos sintomas musculares fica não registrada; e (c) outro problema é a dispersão de sintomas músculo-esqueléticos em uma ampla estratificação de categorias diagnósticas que não são somadas entre si: transtorno muscular, miopatia, rigidez muscular, contração muscular, mialgia, fraqueza muscular, cãibras musculares, fadiga muscular, necrose muscular e espasmo muscular. Somados, o número total de eventos adversos relacionados com miopatia é substancial (18). Além disso, há registros de que ele é bem maior nos prontuários eletrônicos do que nos ensaios clínicos (28), chegando a $25 \%$ ou mesmo $40 \%$ (29). Adicionalmente, a fadiga e a mialgia podem prejudicar a atividade física e a adesão aos tratamentos (30). Relatos de casos de médicos usuários de estatinas revelam impactos significativos dos efeitos adversos musculares (31). Mesmo que seja considerada a estimativa de $10 \%$, como atualmente se estima a incidência desses efeitos adversos (32), isso significa um grande contingente, milhões de pessoas prejudicadas com mínimos benefícios para poucos (12). Outros estudos estimam de 9 a 20\% esta incidência nos usuários de estatinas (27). Parece certo que a incidência e gravidade desses efeitos adversos são maiores do que os ensaios clínicos registram.

Há evidências de que o baixo colesterol está associado a doenças no Sistema Nervo Central (SNC), incluindo transtornos de humor e cognitivo, inclusive com plausibilidade biológica em modelos animais e experimentais humanos. Múltiplas linhas de pesquisa indicam que baixos níveis de colesterol, em geral, e estatinas, em particular, estão associados à neuropatologia e pior funcionamento cognitivo (18). Não se sabe o que se deve às estatinas e o que se deve à baixa do colesterol. Todavia, há divergência entre as evidências $(3,33)$ e plausibilidade para ambos os lados da polêmica $(18,20)$. O colesterol mais baixo é associado com violência e comportamento agressivo, depressão, suicídio, transtornos cognitivos com perda memória e Alzheimer (18). Nos ensaios clínicos com estatinas tais efeitos são considerados raros. Isso ocorre provavelmente porque também aqui há dispersão dos efeitos adversos em muitas categorias. Por exemplo, no FDA Adverse Event Reporting System, há 23 categorias de reação adversa separadas para esse tipo de efeitos adversos (18). Os efeitos sobre - SNC de piora cognitiva costumam aparecer gradualmente e podem ser atribuídos à idade (fator de confusão). Outro agravante, é que os idosos, por terem mais multimorbidade e polifarmácia, merecem mais cautela, e ao mesmo tempo são os que têm maior RCV. Eles têm sabidamente maior 
risco de receber estatinas e também de sofrer de seus efeitos adversos, relacionados ao câncer, à cognição e à dificuldade para exercícios $(26,34)$ exercícios estes que, nos idosos, têm múltiplos efeitos terapêuticos e preventivos benéficos. De fato, nos EUA, a proporção de idosos usando estatinas para P1 com mais de 79 anos aumentou de 8,8\% em 2000 para 34,1\% em 2012 (35). O estudo PROSPER (36), o maior com idosos, não recomenda estatinas acima de 75 anos (37). Para complementar, um mais recente ensaio clínico com idosos não mostrou benefício nenhum no uso de estatina como P1 (38).

Outro efeito adverso consensual é um maior risco de diabetes tipo 2, estimado em um caso novo a cada 50 usuários de estatinas (32). Ele aparece nos ensaios clínicos e em estudos observacionais (39). Também há associação do uso de estatinas com catarata. As estatinas, pela inibição da enzima HMG-CoAR reductase, provoca catarata em modelos animais e há evidências estatísticas observacionais claras dessa relação também em humanos (40). Estudo observacional com 2 milhões de pacientes (30-84 anos), 10\% dos quais novos usuários de estatinas, mostrou que o risco de catarata foi significativamente aumentado dentro de um ano após o início das estatinas, persistiu no tratamento e voltou ao normal no primeiro ano após a interrupção (41). Os ensaios clínicos com estatinas como $\mathrm{P} 1$ relatam efeitos oculares, porém sem atingir significância estatística. Não está claro porque, conhecendo o efeito no cristalino dos inibidores da síntese do colesterol, e havendo evidências observacionais nítidas, tal efeito adverso das estatinas não aparece com relevância estatística nos ensaios (42). A literatura favorável ao uso das estatinas como P1 atribui esses achados a erros, limitações e confundidores dos estudos observacionais.

Em síntese, de 100 pessoas tomando estatina por 5 anos, 2 evitarão um infarto do miocárdio e 98 não terão benefício; sem redução de mortalidade para nenhuma causa. Em contrapartida, 5 a 20 irão sentir dores musculares, fraqueza, fadiga, disfunção cognitiva e aumento do risco de diabetes (17). Vale destacar que os pequenos benefícios se distribuem de forma diferente dos danos: enquanto os pacientes de maior RCV tem mais chance de benefícios, os danos distribuem-se igualmente pelos usuários. Logo, os com menor RCV têm igual potencial de prejuízos com menor benefício (17). E existem também os prejuízos indiretos: os pacientes que usam estatina têm maior chance de se tornarem obesos e mais sedentários do que os não-usuários (43).

Um outro agravante geral dos ensaios clínicos e das revisões e metanálises envolvendo as estatinas como P1 é o sistemático conflito de interesses dos pesquisadores. A quase totalidade deles são financiados pelas indústrias fabricantes das estatinas. Sabe-se que a presença de conflitos de interesse desse tipo gera forte e sistemático viés nos resultados, com superestimação dos benefícios e minimização dos danos (44). Quanto à metanálise da CTT de 2012, todos os autores dos ensaios clínicos metanalisados foram em grande parte ou totalmente comercialmente financiados (11). A própria CTT é parte da Clinical Trial Service Unit of Oxford, que é fortemente dependente de dinheiro das empresas farmacêuticas para suas pesquisas (10). Dos revisores que produziram a diretriz mencionada favorável do NICE, 8 dos 16 também eram financiados pela indústria dos fármacos (45). Tudo o que pensamos que sabemos sobre a eficácia e segurança de estatinas foi produzido por pesquisadores financiados pelos seus fabricantes, cujos interesses comerciais mantêm os dados reais em sigilo (10).

Os exemplos da influência nefasta dos interesses comerciais na produção do conhecimento médico estão fartamente documentados $(46,47)$. No caso das estatinas em $\mathrm{P} 1$, um dos indícios que sugerem desconfiança em relação à idoneidade desses ensaios é a estranha paridade quase perfeita entre efeitos adversos nos braços controle e intervenção nos vários ensaios clínicos, com variações muito grandes da prevalência, a qual não teria porque variar grandemente nos controles.

Em novembro de 2016, a equipe de revisores do CTT publicou no Lancet uma longa argumentação defendendo a eficácia e segurança da estatinas (48), com mais de 300 referências. A argumentação baseia-se integralmente na maior potência dos ensaios clínicos para estabelecer a eficácia e os 
danos das estatinas, bem como nas fragilidades dos estudos observacionais nesse sentido. Entretanto, tal artigo superestimou em dez vezes os benefícios (49), fez extrapolações indevidas (49), unilaterais e parciais quanto a potência dos ensaios clínicos na detecção e mensuração dos danos. Uma discussão mais equilibrada sobre os pontos fortes e fracos de ambos os tipos de estudos encontra-se em Mansi e Mortensen (20), argumentando porque ambos os tipos de estudos são necessários e válidos, têm limitações e devem ser considerados e aperfeiçoados. Os desenhos dos ensaios clínicos, principalmente os financiados pelas indústrias farmacêuticas, são construídos mais para mostrar os benefícios do que os danos (20). No entanto, nenhuma palavra é dedicada ao problema da opacidade e dos conflitos de interesse, nem à reavaliação independente da metanálise de 2012, com resultados contrários aos do $\mathrm{CTT}$. Há forte tendência de consenso científico sobre a necessidade de compartilhamento dos dados entre cientistas para melhorar a confiabilidade das revisões e conclusões (50).

\section{CRITÉRIOS TÉCNICOS E ÉTICOS PARA DECISÃO}

Em P1, embora os quatro princípios ou diretrizes bioéticas clássicas sempre devem estar presentes, na prevenção há predominância da nãomaleficência frente à beneficência $(51,52,53,54)$. No caso das estatinas, que têm grande potencial de medicalização e iatrogenia, como em qualquer medida preventiva com essas características, a atitude inicial e basal dos profissionais deve ser de ceticismo e de resistência à intervenção. Apenas evidências científicas de ótima qualidade e idôneas sobre resultados finais, repetidas, convergentes e consensuais de que os benefícios são amplos frente a mínimos danos, devem vencer a resistência à intervenção, nos convencendo de que vale a pena intervir em pessoas assintomáticas (54).

Para responder a esses rigorosos quesitos, são necessários vários estudos de intervenção de boa qualidade com revisões sistemáticas e metanálises com dados primários disponíveis a pesquisadores independentes, de modo a se garantir a qualidade dos métodos usados e coerências/convergências de resultados (55).

Após a implantação das medidas nas populações, estudos observacionais são necessários e valiosos para se aferir resultados (benéficos e danosos) da intervenção preventiva nas populações reais. Os ensaios clínicos sempre mostram melhores resultados que os obtidos na realidade social das instituições e serviços de saúde, devido a sua artificialidade. Já os estudos observacionais fornecem novos dados, impossíveis ou difíceis de serem obtidos via ensaios clínicos (20).

No caso das estatinas, os resultados quanto aos danos, o ponto em que há grande ignorância, mostraram associação significativa do uso de estatina com miopatia, catarata, diabetes, insuficiência renal aguda e disfunção hepática moderada ou grave (41). Por sua vez, os estudos observacionais mostraram muito maior incidência dos efeitos adversos identificados nos ensaios, e vários outros não identificados, sobre os quais há fortes dúvidas e suspeitas.

O princípio da precaução orienta que devemos evitar danos significativos potenciais antes de eles acontecerem. Ele deve ser aplicado em caso de dúvida científica sobre os danos (ou sobre o balanço danos-benefícios), o que é justamente o caso das estatinas; e por isso não devemos recomendá-las, optando por alternativas inofensivas (55).

\section{CONCLUSÃO}

Descrita sinteticamente a situação da produção das evidências, fica claro que "persiste a necessidade de esclarecer o risco de danos e de benefícios das estatinas" (56). A situação de opacidade dos dados primários e de financiamento pelos fabricantes da quase totalidade dos ensaios clínicos com estatinas para P1 exige grande desconfiança. Para EveryPalmer e Howick (57), aceitar essas evidências seria como permitir aos políticos que contem seus próprios votos, sem fiscalização, e acreditar no resultado.

Uma vez que a nova edição do Tratado está publicada, o novo capítulo sobre P1 com estatinas (7) deve ser problematizado quanto à implícita aceitação da recomendação de uso das estatinas 
como P1. Os pacientes devem ser esclarecidos sobre danos e benefícios e a incerteza dessa recomendação. Se for o caso, sugerimos utilizar a calculadora de risco Best Science Medicine no link (http://chd.bestsciencemedicine.com/calc2.html) para uma decisão compartilhada. Recomendamos que os profissionais e estudantes se apoiem no mesmo capítulo (6) da edição anterior do Tratado.

São grandes os interesses comerciais e pressões quanto ao uso das estatinas, influenciando mesmo as instituições mais respeitadas. Norman et al (58) e a pesada crítica a uma recente revisão Cochrane (59) exemplificam esse contexto. A medicina baseada em evidências não é independente, tem agendas e valores (60) influenciados por interesses específicos e frequentemente escusos $(57,61)$. Ela é uma caixa-preta que precisa ser aberta minimamente, sobretudo quando envolve intervenções preventivas com alto potencial de sobremedicalização e iatrogenia, as quais exigem muita prevenção quaternária (62). Devemos sair de uma postura passiva frente a mudanças de largo alcance clínico e sanitário, que impactam todos, individual e coletivamente, institucional e socialmente, para uma postura mais ativa, crítica e independente. Como afirma Gérvas, estatinas em P1? No gracias (63).

\section{REFERÊNCIAS BIBLIOGRÁFICAS}

(1) Gusso G, Lopes JMC (Orgs.). Tratado de Medicina de Família e Comunidade. 2ed. Porto Alegre: Artmed; 2012 (2 volumes).

(2) Duncan BB et al. Medicina ambulatorial: condutas de atenção primária baseadas em evidências. 4a ed. Porto Alegre: Artmed; 2013.

(3) Zhang X, Wen J, Zhang Z. Statins use and risk of dementia. Medicine, 2018; 97(30), e11304.doi:10.1097/md.0000000000011304

(4) Braga DC et al. Uso de atorvastatina na prevenção primária das doenças cardiovasculares. Arquivos Catarinenses de Medicina, [S.I.], 2016; 44 (4):87-95. Disponível em: <http://www.acm.org.br/acm/seer/index.php/arqui vos/article/view/52>. Acesso em: 07 fev. 2019.
(5) Lama Alexis. ¿Estatinas en Prevención Primaria?. Rev Chil Cardiol. 2010; 29(3): 799-380. http://dx.doi.org/10.4067/S071885602010000300019 .

(6) Justino AL, Calvette TF, Melo, TAP. P1 e secundária para doenças cardiovasculares. In: Gusso G, Lopes JMC (Orgs.). Tratado de Medicina de Família e Comunidade. 1a ed. Porto Alegre: Artmed, 2012 , v. I, p. $1233-1238$

(7) Liu, GKH, Silva BLS. P1 e secundária para doenças cardiovasculares. In: Gusso G, Lopes JMC, Dias LC. (Orgs.). Tratado de Medicina de Família e Comunidade. 2aed.Porto Alegre: Artmed, 2018, v. 1, p. 1334-1342.

(8) Cholesterol Treatment Trialists' (CTT) Collaborators. The effects of lowering LDL cholesterol with statin therapy in people at low risk of vascular disease: meta-analysis of individual data from 27 randomised trials. Lancet 2012; 380:581590. doi: 10.1016/S0140-6736(12)60367-5.

(9) Taylor F, Huffman MD, Macedo AF et al. Statins for the primary prevention of cardiovascular disease. Cochrane Database Syst Rev 2013;1:CD004816.

(10) Abramsom J. Prescribing statins: time to rein it in. Pharm J. 2015; 294. doi:10.1211/PJ.2015.20068145.

(11) Abramson J, Rosenberg HG, Jewell $\mathrm{N}$ et al. Should people at low risk of cardiovascular disease take a statin? The BMJ 2013;347:f6123. doi: 10.1136/bmj.f6123

(12) Fiona G. Adverse effects of statins BMJ 2014;348 :g3306. https://www.bmj.com/content/348/bmj.g3306

(13) National Clinical Guideline Centre (UK) Lipid modification: cardiovascular risk assessment and the modification of blood lipids for the primary and secondary prevention of cardiovascular disease. London: National Institute for Health and Care Excellence (UK); 2014. https://www.ncbi.nlm.nih.gov/books/NBK248067/ Acesso em 06 Jan 2019. 
(14) Stone NJ, Robinson JG, Lichtenstein AH et al. $2013 \mathrm{ACC} / \mathrm{AHA}$ guideline on the treatment of blood cholesterol to reduce atherosclerotic cardiovascular risk in adults: a report of the American College of Cardiology/American Heart Association Task Force on Practice Guidelines. Circulation 2014;129(2):S1-S45.

(15) Pulse - At the heart of general practice since 1960. http://www.pulsetoday.co.uk/yourpractice/qof/nice-recommends-introduction-of-10statin-threshold-into-qof-despite-gpopposition/20010115.article\#.VcczhpPtmko. Acesso em 04 Jan 2019.

(16) Cholesterol Treatment Trialists' (CTT) Collaboration. Protocol for a prospective collaborative overview of all current and planned randomized trials of cholesterol treatment regimens. Am J Cardiol. 1995; 75(16):1130-1134.

(17) Redberg RF, Katz MH. Statins for Primary Prevention. JAMA 2016; 316(19), 1979. doi:10.1001/jama.2016.15085

(18) Diamond DM, Ravnskov U. How statistical deception created the appearance that statins are safe and effective in primary and secondary prevention of cardiovascular disease, Expert Rev Clin Pharmacol 2015; 8(2):201-210. doi: 10.1586/17512433.2015.1012494

(19) Kristensen ML, Christensen PM, Hallas J. The effect of statins on average survival in randomised trials, an analysis of end point postponement. BMJ Open. 2015; 5(9):e007118.

(20) Mansi I, Mortensen E. The controversy of a wider statin utilization: why? Expert Opin Drug Saf 2013; 12(3),327-37. doi:10.1517/14740338.2013.779667

(21) Mascitelli L, Goldstein MR. Comment on 'Statin use and all-cancer survival: prospective results from the Women's Health Initiative'. Br J Cancer. 2017;116(3):e1-e1. doi:10.1038/bjc.2016.395.

(22) Benn M, Tybjærg-Hansen A, Stender S, Frikke-Schmidt R, Nordestgaard BG. Low-density lipoprotein cholesterol and the risk of cancer: a mendelian randomization study. J Natl Cancer Inst 2011; 103(6): 508-519.

(23) Alsheikh-Ali AA, Trikalinos TA, Kent DM, Karas RH (2008) Statins, low-density lipoprotein cholesterol, and risk of cancer. J Am Coll Cardiol 52(14): 1141-1147.

(24) McDougall JA, Malone KE, Daling JR, et al. Long-term statin use and risk of ductal and lobular breast cancer among women 55 to 74 years of age. Cancer Epidemiol Biomarkers Prev 2013; 22:152937. doi: 10.1158/1055-9965.EPI-13-0414

(25) Wang A, Aragaki AK, Tang JY, et al. Statin use and all-cancer survival: prospective results from the Women's Health Initiative. $\mathrm{Br} J$ Cancer. 2016;115(1):129-35.

(26) Mangin D, Sweeney K, Heath I. Preventive health care in elderly people needs rethinking. BMJ 2007; 335(7614):285-287. doi: 10.1136/bmj.39241.630741.BE1

(27) Fernandez G, Spatz ES, Jablecki C, Phillips PS. Statin myopathy: a common dilemma not reflected in clinical trials. Cleve Clin J Med 2011;78(6):393-403. doi: 10.3949/ccjm.78a.10073

(28) Yerrakalva D, Griffin SJ. Statins for primary prevention in people with a 10\% 10-year cardiovascular risk: too much medicine too soon? British J Gen Pract 2017; 67(654):40-41. https://doi.org/10.3399/bjgp17X688789

(29) Golomb BA, Evans MA, Dimsdale JE, et al. Effects of statins on energy and fatigue with exertion: results from a randomized controlled trial. Arch Intern Med 2012;172:1180-2. doi: 10.1001/archinternmed.2012.2171

(30) Opie LH. Exercise-induced myalgia may limit the cardiovascular benefits of statins. Cardiovasc Drugs Ther; 2013; 27(6):569-572. doi: 10.1007/s10557-013-6483-8

(31) Koslik HJ, Meskimen AH, Golomb BA. Physicians' 39; Experiences as Patients with Statin Side Effects: A Case Series. Drug Saf Case Rep 2017; 4(1):3. doi: 10.1007/s40800-017-0045-0

(32) The NNT. http://www.thennt.com/nnt/statins-for-heart- 
disease-prevention-without-prior-heart-disease-2/ Acesso em 03 Jan 2019.

(33) Chu C-S, Tseng P-T, Stubbs B, et al. Use of statins and the risk of dementia and mild cognitive impairment: a systematic review and meta-analysis. Sci Rep. 2018; 8:5804.

(34) Scondotto G, Pojero F, Addario SP et al. The impact of polypharmacy and drug interactions among the elderly population in Western Sicily, Italy. Aging Clin Exp Res (2018) 30: 81. https://doi.org/10.1007/s40520-017-0755-2.

(35) Johansen ME, Green LA. Statin use in very elderly individuals, 1999-2012. JAMA Intern Med. 2015; 175(10):1715-1716. doi: 10.1001/jamainternmed.2015.4302

(36) Shepherd J, BlauwGJ, MurphyMB, Bollen EL, Buckley BM, Cobbe SM, et al. Pravastatin in elderly individuals atriskof vascular disease (PROSPER): a randomised controlled trial. Lancet 2002;360:1623-30

(37) Papadakis MA, McPhee SJ, Rabow MW (editors). Current Medical Diagnosis \& Treatment. New York: McGraw-Hill Education; 2019.

(38) Han BH, Sutin D, Williamson JD, Davis BR, Piller LB, Pervin, H. Effect of Statin Treatment vs Usual Care on Primary Cardiovascular Prevention Among Older Adults. JAMA Internal Medicine, 2017; 177(7):955. doi:10.1001/jamainternmed.2017.1442.

(39) Bernardi A, Rocha VZ, Faria-Neto JR. Use of statins and the incidence of type 2 diabetes mellitus. Rev. Assoc. Med. Bras. 2015; 61(4):375$380 . \quad$ http://dx.doi.org/10.1590/18069282.61.04.375.

(40) Machan CM, Hrynchak PK, Irving EL. Agerelated cataract is associated with type 2 diabetes and statin use. Optom Vis Sci 2012; 89:1165-71.

(41) Hippisley-Cox H, Coupland C. Unintended effects of statins in men and women in England and Wales: population based cohort study using the QResearch database, BMJ 2010;340:c2197 https://doi.org/10.1136/bmj.c2197

(42) Ferrer Jl. Estatinas, uso racional en el tratamiento de la dislipoproteinemia. Rev Cubana
Med Gen Integr 2009; 25(2). http://scielo.sld.cu/scielo.php?script=sci_arttext\&pi d=S0864-21252009000200009\&Ing=es. Acessado em 04 Jan 2019.

(43) Sugiyama T, Tsugawa $\mathrm{Y}$, Tseng C-H, Kobayashi Y, Shapiro MF. Different time trends of caloric and fat intake between statin users and nonusers among US adults: gluttony in the time of statins? JAMA Intern Med. 2014;174(7):1038-1045. doi: 10.1001/jamainternmed.2014.1927

(44) Lundh, A., Lexchin, J., Mintzes, B., Schroll, J. B., Bero, L. Industry sponsorship and research outcome. Cochrane Database of Systematic Reviews. 2017. doi:10.1002/14651858.mr000033.pub3

(45) Fiable: Información en salud. http://informacionfiabledesalud.com/?p=5630. Acesso em 05 Jan 2019.

(46) Gøtzsche PC. Deadly Medicines and Organised Crime: How Big Pharma Has Corrupted Healthcare. London: Radcliffe Publishing; 2013.

(47) Angel M. A verdade sobre os laboratórios farmacêuticos: como somos enganados e o que podemos fazer a respeito. 2a edição. Rio de Janeiro, Record; 2007.

(48) Collins C, Reith C, Emberson J et al. Interpretation of the evidence for the efficacy and safety of statin therapy. Lancet 2016; 388:25322561. doi: 10.1016/S0140-6736(16)31357-5

(49) Abramson J, Rosenberg HG, Jewell N, Wright JM. Safety and efficacy of statins. Lancet 2017; 357:1097. doi:10.1016/S01406736(17)30713-4.

(50) Academy of Medical Sciences. Enhancing the use of scientific evidence to judge the potential benefits and harms of medicines. 2017 https://acmedsci.ac.uk/file-download/44970096. Acesso em 06 Jan 2019.

(51) Segura A. Inducción sanitaria de los cribados: impacto y consecuencias. Aspectos éticos. Gac Sanit 2006; 20 (Supl 1): 88-95. https://doi.org/10.1157/13086031 
(52) Weingarten $M$, Matalon A. The ethics of basing community prevention in general practice. $J$ Med Ethics 2010; 36:138-141. doi: 10.1136/jme.2009.032284

(53) Rose, G. Estratégias da medicina preventiva. Porto Alegre: Artmed; 2010.

(54) Tesser CD, Norman AH. Differentiating clinical care from disease prevention: a prerequisite for practicing quaternary prevention. Cad. Saúde Pública. 2016; 32(10):e00012316. doi: 10.1590/0102-311X00012316

(55) Tesser CD, Norman AH. Geoffrey Rose e o princípio da precaução: para construir a prevenção quaternária na prevenção. Interface (Botucatu) 2019, no prelo.

(56) Yerrakalva D, Griffin SJ. Statins for primary prevention in people with a 10\% 10-year cardiovascular risk: too much medicine too soon? $\mathrm{Br}$ J Gen Pract 2017; 67(654): 40-41. doi: 10.3399/bjgp17X688789

(57) Every-Palmer S,.Howick J. How evidencebased medicine is failing due to biased trials and selective publication. J Eval Clin Pract, 2014; 20(908914). doi: 10.1111/jep.12147
(58) Norman AH, Hunter DJ, Russell AJ. Linking high-risk preventive strategy to biomedical-industry market: implications for public health. Saude soc. 2017; 26(3): 638-650. http://dx.doi.org/10.1590/s0104-12902017172682

(59) Jørgensen L, Gøtzsche PC, Jefferson T. The Cochrane HPV vaccine review was incomplete and ignored important evidence of bias. BMJ EvidenceBased Medicine 2018;23:165-168.

(60) Kelly MP, Heath I, Howick J, et al. The importance of values in evidence-based medicine. BMC Med Ethics. 2015;16(1):69. doi:10.1186/s12910-015-0063-3

(61) Greenhalgh T, Howick J, Maskrey N. Evidence based medicine: a movement in crisis? BMJ 2014; 348:g3725. https://doi.org/10.1136/bmj.g3725

(62) Norman AH, Tesser CD. Quaternary prevention: a balanced approach to demedicalisation. Br J Gen Pract. 2019; 69(678):2829. doi:10.3399/bjgp19X700517

(63) http://www.nogracias.eu/2016/10/04/urgentelas-estatinas-no-salvan-vidas/. Acesso em 16 Jan 2019.

\section{ABSTRACT}

This article reviews the changes in the attitude and medical practices regarding the use of statins as primary prevention (P1). This issue is of great clinical relevance for Brazilian primary care and public health alike. In the Treaty of Family and Community Medicine, 2018 edition, the chapter on primary prevention of cardiovascular disease implicitly assumes that statins should be used as P1 without any discussion of the problems involved in this recommendation. This article summarizes the problems and controversies involved in the prescription of statins in P1, from 2012 onwards when a first meta-analysis recommended its use. We critically discuss the evidence on statins from classic ethical and technical criteria. Wdse contend that evidence to use statins as P1 is weak and unreliable, in the face of significant potential harms and very small benefits. More research is needed, more transparent and less pharmaceutical influenced-laden.

Keywords: Primary Health Care; Primary Prevention; Quaternary Prevention; Cardiovascular Diseases; Cardiovascular Agents. 